\title{
Práticas de saúde mental na Atenção Primária à Saúde: percepções de trabalhadores
}

\author{
Mental health practices in Primary Health Care: workers' perceptions \\ Prácticas de salud mental en la Atención Primaria de Salud: percepciones de los trabajadores
}

Recebido: 04/02/2021 | Revisado: 11/02/2021 | Aceito: 15/02/2021 | Publicado: 22/02/2021

\author{
Paolo Porciúncula Lamb \\ ORCID: https://orcid.org/0000-0002-6712-0168 \\ Universidade Federal da Paraíba, Brasil \\ E-mail: paoloplamb@gmail.com \\ Geraldo Eduardo Guedes de Brito \\ ORCID: https://orcid.org/0000-0002-3059-3164 \\ Universidade Federal da Paraíba, Brasil \\ E-mail: eduardo.guedes.ufpb@gmail.com \\ Andréa Loureiro Roges \\ ORCID: https://orcid.org/0000-0003-0109-2114 \\ Secretaria da Saúde de Recife, Brasil \\ E-mail: deiaroges@hotmail.com \\ Cora Coralina dos Santos Junqueira \\ ORCID: https://orcid.org/0000-0002-1105-4974 \\ Universidade Federal da Paraíba, Brasil \\ E-mail: coracoralina33@gmail.com \\ Robson da Fonseca Neves \\ ORCID: https://orcid.org/0000-0002-3889-560X \\ Universidade Federal da Paraíba, Brasil \\ E-mail: robsonfisioba@gmail.com \\ Sara Virna Alves Barros \\ ORCID: https://orcid.org/0000-0003-2548-6951 \\ Universidade Federal da Paraíba, Brasil \\ E-mail: saravirna@hotmail.com \\ Augusto José Bezerra de Andrade \\ ORCID: https://orcid.org/0000-0003-1406-3558 \\ Universidade Federal da Paraíba, Brasil \\ E-mail: andrade.augustojose@ hotmail.com
}

\begin{abstract}
Resumo
A Estratégia Saúde da Família (ESF) é um dos principais pilares para a organização da Atenção Primária à Saúde no Brasil e vem se tornando um dispositivo importante na produção do cuidado em Saúde Mental. O estudo teve como objetivo conhecer a produção do cuidado em saúde mental por Equipes de Saúde da Família em Recife, capital do estado de Pernambuco, região nordeste do Brasil. Trata-se de um estudo descritivo-exploratório, com abordagem qualitativa. Participaram do estudo 116 enfermeiros da ESF através de um questionário online e 11 trabalhadores de três equipes de uma Unidade de Saúde da Família que participaram de uma entrevista semiestruturada. Os dados foram analisados por meio da Análise de Conteúdo, modalidade temática, proposta por Bardin. Evidenciou-se que as principais ações em saúde mental relatadas pelos participantes foram: consultas médicas, tratamento medicamentoso, grupos terapêuticos e visitas domiciliares. Entre as potencialidades no cuidado em saúde mental na atenção primária destacaram-se o apoio matricial, o projeto terapêutico singular, a criação de vínculos e as práticas integrativas e complementares. Observaram-se como fragilidades a restrita oferta de educação permanente e a desarticulação da Rede de Atenção Psicossocial. Concluiu-se que a ESF contribui para a produção do cuidado em saúde mental desenvolvida na realidade estudada e aproxima-se de práticas em saúde mental de base territorial e comunitária, representando uma ruptura no modelo manicomial. Ademais, constatou-se que as ações acolhedoras qualificam a relação profissional-usuário.
\end{abstract}

Palavras-chave: Assistência à saúde mental; Saúde mental; Atenção primária à saúde; Conhecimentos, atitudes e práticas em saúde.

\footnotetext{
Abstract

The Family Health Strategy (FHS) is one of the main pillars for the organization of Primary Health Care in Brazil and has become an important device in the production of mental health care. The study aimed to know the production of mental health care by Family Health Teams in Recife, capital of the state of Pernambuco, in the northeast from Brazil. This is a descriptive-exploratory study, with a qualitative approach. 116 nurses from the FHS nurses participated in the study through an online questionnaire, and 11 workers from three teams from a Family Health Unit participated in a semi-structured interview. The data were analyzed using Content Analysis, thematic modality, proposed by Bardin.
} 
It was evidenced that the main mental health actions reported by the participants were: medical consultations, pharmacological treatment, therapeutic groups and home visits. Among the potentialities of mental health care in primary care, the matrix support, singular therapeutic project, the creation of bonds, and integrative and complementary practices were highlighted. It was observed as weaknesses the limited offer of permanent education and the disarticulation of the Psychosocial Care Network. It was concluded that the FHS contributes to the production of mental health care developed in the studied reality, and is close to the territorial- and community-based mental health practices, representing a rupture in the manicomial model. Furthermore, it was found that supportive actions qualify the professional-user relationship.

Keywords: Mental health assistance; Mental health; Primary health care; Health knowledge, attitudes, practice.

\section{Resumen}

La Estrategia de Salud de la Familia (ESF) es uno de los pilares principales la organización de la Atención Primaria de Salud en Brasil y se ha convertido en un dispositivo importante en la producción de atención de salud mental. El estudio tuvo como objetivo conocer la producción de atención en salud mental por los Equipos de Salud de la Familia en Recife, capital del estado de Pernambuco, en el noreste de Brasil. Este es un estudio descriptivo-exploratorio, con enfoque cualitativo. 116 enfermeros de la ESF participaron del estudio a través de un cuestionario online y 11 trabajadores de tres equipos de una Unidad de Salud de la Familia que participaron en una entrevista semiestructurada. Los datos fueron analizados mediante Análisis de Contenido, modalidad temática, propuesta por Bardin. Se evidenció que las principales acciones de salud mental informadas por los participantes fueron: consultas médicas, tratamiento farmacológico, grupos terapéuticos y visitas domiciliarias. Entre las potencialidades de la atención en salud mental en la atención primaria se destacarons oporte matricial, proyecto terapéutico singular, la creación de vínculos y prácticas integrativas y complementarias. Se observaron debilidades en la oferta limitada de educación permanente y en la desarticulación de la Red de Atención Psicosocial. Se concluyó que la ESF contribuye a la producción de atención en salud mental desarrollada en la realidad estudiada y se acerca a las prácticas territorial y comunitaria de salud mental, lo que representa una ruptura en el modelo manicomial. Además, se encontró que las acciones de apoyo califican la relación profesional-usuario.

Palabras clave: Atención a la salud mental; Salud mental; Atención primaria de salud; Conocimientos, actitudes y prácticas en salud.

\section{Introdução}

De acordo com a Organização Mundial de Saúde (OMS), um em cada dez indivíduos no mundo possui algum transtorno mental. Globalmente, o sofrimento psíquico, os transtornos mentais, neurológicos e o uso de substâncias psicoativas vêm apresentando um alto e crescente índice de prevalência, representando grande carga de adoecimento (OMS, 2016). No Brasil, os transtornos mentais correspondem a aproximadamente $36,5 \%$ das principais causas de incapacidade e morte (OPAS, 2018a). Assim, abordagem das questões relacionadas à Saúde Mental (SM) configura-se, atualmente, como um desafio para os sistemas de saúde.

No que se refere à organização dos sistemas de saúde, há evidências que aqueles com uma Atenção Primária à Saúde (APS) estruturada se mostram mais eficientes, resolutivos e ampliam o acesso à assistência de saúde para a população (Giovanella et al., 2019). O fortalecimento deste nível de atenção é incentivado pela OMS, que reconhece a APS como um nível de assistência que atua na promoção de condições de saúde e de desenvolvimento humano mais equitativo. O Plano de Ação em Saúde Mental 2015-2020 da Organização Panamericana da Saúde (OPAS) tem com um de seus objetivos a ampliação dos serviços de base comunitária e as ações em SM na APS, uma vez que se reconhece o potencial deste nível de atenção (OPAS, 2018b; Rodriguez, 2010).

No Brasil, a APS expandiu-se, por meio da Estratégia Saúde da Família (ESF), que prioriza o contato preferencial dos usuários, inclusive dos que necessitam dos cuidados associados ao sofrimento psíquico e aos agravos decorrentes ao uso abusivo de álcool e outras drogas no campo da SM, além de ser considerado, o centro de comunicação com toda a Rede de Atenção à Saúde - RAS (Brasil, 2017, 2013). A ESF tem importância capital para a RAS devido à extensão de sua cobertura e facilidade de acesso, continuidade das ações, trabalho em equipe multiprofissional e sua elevada resolutividade (Serapione \& Tesser, 2019), mostrando-se coerente com o proposto pela OMS. 
Com a criação da Rede de Atenção Psicossocial (RAPS) no Sistema Único de Saúde (SUS) em 2011, buscou-se fortalecer a articulação e integração dos seus diversos equipamentos constituintes: APS, atenção psicossocial especializada, atenção de urgência e emergência, atenção residencial de caráter transitório, atenção hospitalar, estratégias de desinstitucionalização e reabilitação psicossocial (Brasil, 2011). De acordo com Moreira e Onocko-Campos (2017), essa configuração da RAPS, no que se refere à atenção e à produção do cuidado em SM, favorece a atuação das equipes de saúde inseridas na APS, permitindo trocas de saberes entre os trabalhadores e maior articulação da rede de serviços, tendo em vista o cuidado ampliado para as necessidades dos usuários.

Nesse contexto, as equipes de Saúde da Família (EqSF) possuem em seu cotidiano de trabalho expressivas demandas do campo da SM. Porém, de acordo com Gryschek e Pinto (2015), elas "nem sempre se sentem capazes de lidar com a demanda de SM ou se focam em ações que perpetuam a lógica centrada no cuidado médico especializado e na terapêutica medicamentosa". Porém, esses mesmos autores destacam que "as EqSF, tem plenas condições de desenvolver e ofertar cuidados integrais em SM para os indivíduos e famílias sob sua responsabilidade".As EqSF conhecem as histórias de vida dos usuários adscritos, seus vínculos e o território onde moram (Brasil, 2017).Essa reorientação do modelo de assistência operacionalizada pela ESF, através da abordagem do indivíduo em seu contexto familiar e comunitário, fortalece os laços de comprometimento entre profissionais da ESF, usuários e famílias (Brito, Mendes \& Santos Neto, 2017).

Evidências apontam resultados positivos na oferta de ações em SM por profissionais da APS, que incluem o diagnóstico precoce e acompanhamento de usuários, mostrando-se acessíveis e eficientes (Luitel et al., 2020). Essa temática já foi abordada por alguns estudos realizados no contexto da ESF (Barros et al., 2019; Maia, 2020; Souza, Amarante \& Abrahão, 2019). Entretanto, ainda são persistentes as barreiras para a efetivação do cuidado em SM na APS globalmente (Baker \& Naidu, 2020), que é fortemente indicado também em estudos na ESF (Wenceslau \& Ortega,2015).

Logo, estudos sobre esta temática podem permitir uma análise aprofundada da realidade das práticas em SM na APS, contribuindo para uma melhor compreensão de suas potencialidades e fragilidades. A partir do exposto, o objetivo deste estudo foi conhecera produção do cuidado em SM pela ESF de Recife, capital de Pernambuco, região nordeste do Brasil.

\section{Metodologia}

Trata-se de um estudo descritivo-exploratório, com abordagem qualitativa, desenvolvido em Recife, capital do estado de Pernambuco, região nordeste do Brasil. O processo de coleta dos dados foi operacionalizado por meio de duas etapas: (1) aplicação de instrumento online com enfermeiros atuantes em EqSF de Recife, durante o mês de janeiro de 2019, e (2) entrevistas semiestruturadas com trabalhadores de três EqSF referência do município (médicos, enfermeiros, cirurgiões dentistas e agentes comunitários de saúde - ACS), ocorridas no período durante o mês de fevereiro de 2019.

Para a coleta de dados online foi elaborado um instrumento na plataforma digital Google Forms composto por um formulário contendo variáveis sociodemográficas (sexo, idade, ano de experiência na ESF, tempo de atuação na ESF do município) e três questões abertas que investigavam: (a) quais ações de SM as EqSF dos entrevistados desenvolviam; (b) quais as principais potencialidades para o desenvolvimento de ações no campo da SM na ESF e; (c) quais as principais fragilidades para o desenvolvimento de ações no campo da SM na ESF. Optou-se por aplicá-lo aos enfermeiros uma vez que, a atuação desse profissional norteia-se pelo exercício do gerenciamento de equipes, bem como a assistência e a educação em saúde orientada pelo cuidado biopsicossocial (Balsanelli \& Cunha, 2006; Melo et al.,2013). Assim, avaliou-se que estes trabalhadores possuiriam uma visão ampliada do conjunto de ações desenvolvidas por sua equipe. Para acessar o instrumento online os participantes deveriam realizar a leitura do termo de consentimento e aceitar em participar do estudo.

O convite dos participantes da etapa online foi realizado via e-mail, por meio de uma lista com os endereços eletrônicos dos 276 enfermeiros atuantes na ESF de Recife em dezembro de 2018. Destes 276 e-mails enviados, 132 não foram 
respondidos e 28 não foram entregues aos destinatários. A amostra foi constituída por 116 enfermeiros atuantes na ESF de Recife.

Para a etapa de coleta de dados presenciais foram realizadas entrevistas semiestruturadas individuais com 11 trabalhadores da Unidade de Saúde da Família - USF (três EqSF), buscando aprofundar a compreensão do tópico deste estudo. $\mathrm{O}$ roteiro de entrevista desta fase baseou-se nos mesmos elementos que orientaram as perguntas online, com vistas a aprofundar a compreensão dos elementos emergentes na primeira fase de coleta.

A escolha da USF ocorreu por conveniência, tendo em vista o reconhecimento das ações no campo da SM dessas EqSF. Para a definição do número de participantes para as entrevistas foi adotado o critério de saturação das informações (Saunders et al., 2018). Primeiramente, foi realizado o convite aos trabalhadores e marcação do dia e horário, em seguida, foram realizadas as entrevistas pelo pesquisador principal, com duração média de 60 minutos cada, em uma sala separada, respeitando a privacidade do participante. Houve treinamento piloto para ajustes e familiarização para a condução da entrevista. O pesquisador não possuía nenhuma relação prévia com os participantes do estudo.

As respostas das três questões do instrumento online foram transportadas da planilha Excel para um documento Word. Todas as entrevistas foram audiogravadas, transcritas e submetidas à validação (leitura das transcrições simultaneamente à audição das gravações para identificação dos possíveis erros da transcrição). O material coletado foi submetido à análise de conteúdo na modalidade temática, seguindo os seguintes processos preconizados por Bardin (2011): a) a pré-análise do conteúdo; b) a exploração do material ou codificação; e c) o tratamento dos resultados, a inferência e a interpretação.

Este estudo respeitou a Resolução 466/12 do Conselho Nacional de Saúde (Brasil, 2012), sendo aprovada por Comitê de Ética em Pesquisa com parecer no. 2.924.765 (CAAE: 94108918.8.0000.5188). Todos os participantes forneceram o consentimento para participar do estudo antes do início da coleta dos dados. As entrevistas presenciais foram realizadas em ambiente privativo e foi garantido o anonimato dos participantes. Os profissionais da USF foram identificados pela letra "P" e os enfermeiros do questionário online pela letra "E” e numerados aleatoriamente.

\section{Resultados e Discussão}

Alcançou-se uma taxa de resposta de 48,3\%(n=116) do questionário online. Dos respondentes, 94,8\% eram mulheres, com média de idade de 43,6 anos $(d p=6,9), 80,9 \%$ possuíam mais que dez anos de experiência na ESF, 43,0\% atuavam na ESF deste município há pelo menos dez anos. Dos profissionais da USF, 81,8\% eram mulheres, com média de idade de 48 anos, com tempo médio de atuação na ESF de 13,5 anos. Em relação a categoria profissional dos participantes da entrevista semiestruturada, os entrevistados eram médicos $(n=3)$, enfermeiros $(n=2)$, dois cirurgiões-dentistas $(n=2)$, e ACS ( $n=4)$.

Os resultados foram apresentados com base na descrição e caracterização das práticas, bem como nas categorias prédefinidas: A primeira caracterizava as práticas em SM na ESF; a segunda era composta por fatores positivos e potencializadores no cuidado em SM na ESF e; a terceira indicou as barreiras a serem superados para a melhoria do cuidado em SM na ESF. Estas categorias são apresentadas e discutidas a seguir.

\subsection{A produção do cuidado em saúde mental}

A maioria dos participantes deste estudo afirmou que são desenvolvidas ações de SM em suas EqSF. O acompanhamento individual dos usuários adscritos com algum sofrimento psíquico foi caracterizado pela oferta de consultas médicas, geralmente semanais. Frequentemente tinham como demanda a prescrição ou transcrição de psicotrópicos e a realização dos encaminhamentos para outros serviços da RAPS. 
Horário de um turno semanal reservado para atendimento individual de SM, com o objetivo de acompanhamento do paciente, troca de receitas, encaminhamento. Ele é feito pelo médico (E13).

Assim, minhas consultas são sempre, agendadas no dia e são atendidos no mesmo dia, vem sempre com essa solicitação de renovação de receita (P1).

Evidências confirmam que a expansão da ESF impactou positivamente em diversas dimensões das condições de saúde da população brasileira, inclusive, favorecendo o acesso a consultas médicas (Macinko \& Mendonça, 2018). No caso específico do campo da SM, elas já se encontram como uma ação programada entre as equipes deste estudo. Tal resultado pode ser considerado um avanço, uma vez que, historicamente, esta ação ocorria principalmente nos níveis secundário e terciário, ou seja, em clínicas e hospitais psiquiátricos (Amarante \& Nunes, 2018; Moliner \& Lopes, 2013).

Além disso, as consultas médicas podem funcionar como coordenadoras do cuidado na RAPS ao qualificarem os encaminhamentos. Porém, alguns profissionais da APS podem perceber a ampliação da oferta de cuidados em SM neste nível de atenção como sobrecarga de trabalho e um "desafogamento" da Atenção Especializada (Gryschek \& Pinto, 2015). Assim, torna-se importante que todos os profissionais da RAPS tenham clareza de seu papel no cuidado em saúde mental.

Contudo, ao qualificarem a função destas consultas individuais, evidenciou-se que elas se destinavam, na maioria dos casos, à prescrição e transcrição de medicamentos psicotrópicos. Com isso, percebeu-se a persistência de uma prática medicalizadora em detrimento daquelas que consideram a clínica ampliada, trabalho em rede, e prevenção de complicações e promoção da saúde. Tal situação é uma realidade que envolvem profissionais e usuários, ocorrendo para além de uma assistência à saúde biomédica, na qual a prescrição do psicotrópico é o foco, a população precisa compreender a importância de uma assistência humanizada e a continuidade do cuidado com outras práticas de saúde para alcançar um bem estar físico, mental e social.

Há muitos usuários fazendo uso de psicotrópicos, sem o devido acompanhamento, simplesmente vão para unidade de saúde renovar receita (E66).

A gente faz o acolhimento né, no acolhimento eles procuram a gente, a enfermeira para conversar sobre. É mais para pedir para médica renovar a receita da medicação (P7).

Frequentemente, a ESF acaba reproduzindo o modelo psiquiátrico tradicional, biomédico, através de práticas curativistas e mecadilizadoras, se contrapondo a nova proposta de cuidado, cujas ações de saúde estão centradas no indivíduo, família e comunidade, considerando suas singularidades e aspectos socioculturais (Neto, 2017; Paulon et al., 2013). A Política Nacional de Saúde Mental compreende as intervenções da APS como fundamentais no cuidado da pessoa com sofrimento psíquico, possibilitando a superação da medicalização enraizada na sociedade e a patologização do indivíduo. Para isso, baseado na compreensão da clínica ampliada, a produção do cuidado é compartilhada entre os diversos profissionais, propondo novas possibilidades deste cuidado para além do tratamento medicamentoso (Brasil, 2017; Tesser \& Campos, 2010; Zanella et al., 2016). Portanto, considerar a singularidade de cada indivíduo é fundamental, pois dessa maneira o profissional poderá oferecer uma assistência baseada no diálogo a fim de tornar o usuário sujeito do seu cuidado.

A segunda prática mais recorrente apontada foram os grupos de SM. Em sua maioria, apresentavam caráter interprofissional, realizados em parceria com o Núcleo Ampliado de Saúde da Família e Atenção Básica (Nasf-AB) e em algumas situações, durante atividades de ensino de cursos de residência em saúde. Os grupos tinham periodicidade semanal, 
quinzenal ou mensal, com a utilização de metodologias e atividades diversas, como, por exemplo, a prática de exercícios físicos, a educação em saúde, roda de conversa, palestras, escuta qualificada e terapia comunitária.

Grupo de 15 em 15 dias na unidade, com palestras, passeios, exercícios, envolvendo todos os profissionais que queiram participar (E1).

Temos um grupo de SM, com a participação do Nasf-AB e seus residentes, com reunião semanal (E4).

[...] tem o grupo Bem Viver, que é da psicóloga do Nasf-AB, toda terça-feira às 14 horas [...] tem um grupo que é encaminhado por nós médicos, enfermeiros, alguém da comunidade ou próprio agente de saúde (P3).

Neste estudo, as atividades em grupo se configuraram como práticas interprofissionais na ESF. O trabalho interprofissional caracteriza-se como uma prática profissional potente para otimizar as habilidades dos trabalhadores de saúde, na perspectiva de trocas e compartilhamento de saberes, fortalecendo assim o cuidado em saúde centralizado no usuário e família (Peduzzi, 2013). Com isso, espera-se melhorar a resolutividade, a continuidade, a coordenação do cuidado e a tomada de decisões de forma compartilhada contribuindo para a mudança do modelo de atenção e processo de trabalho (Matuda et al., 2015; OMS, 2010; Wetzel et al., 2018).

Os grupos possibilitam a troca de saberes, tanto dos profissionais, quanto dos usuários, principalmente aqueles em sofrimento psíquico - historicamente excluídos das relações sociais no cotidiano - contribuindo para a autonomia e fortalecimento do estabelecimento de vínculos. Corroborando com os resultados deste estudo, Frateschini e Cardoso (2016) referem que as práticas em grupos se destacam pelo fortalecimento de vínculos e confiança entre os participantes e profissionais, através do compartilhamento das experiências vividas. Essas atividades em grupos são importantes ferramentas terapêuticas realizadas na APS, coerentes com o modelo psicossocial, incentivando a participação ativa das pessoas com sofrimento psíquico e contribuindo com o protagonismo e autonomia desses sujeitos (Minozzo et al., 2012).

Outra estratégia evidenciada pelos trabalhadores participantes da pesquisa foram as visitas domiciliares. Esse recurso possibilita a busca ativa e identificação precoce de sofrimento psíquico e transtornos mentais entre a população adscrita pelas EqSF, protagonizadas, principalmente, pelo ACS. Permitiam ainda, o acompanhamento de casos específicos.

Visita domiciliar do ACS mensal e da equipe, em caso de intercorrência ou falta de adesão (E39).

Além disso, o próprio agente de saúde que é o elo da gente na comunidade ele sempre passa e me traz, quais as dificuldades, o que realmente tá precisando, e aí a gente faz uma visita ou a gente abre espaço para o atendimento na própria unidade (P6).

As visitas domiciliares são fundamentais para o cuidado, permitindo uma aproximação dos profissionais ao contexto e dinâmica familiar e comunitária que os usuários estão inseridos, principalmente em casos de pessoas com sofrimento psíquico, que muitas vezes estão isoladas e sensíveis à convivência social. Ela oportuniza conhecer aspectos subjetivos do indivíduo e da família a partir do acolhimento, diálogo e confiança (Andrade et al., 2014).

O profissional ACS destaca-se nesta ação, desenvolvendo o acompanhamento às famílias e indivíduos, o que possibilita um processo crítico e reflexivo no trabalho da equipe. Ademais, para casos específicos, as visitas domiciliares 
demandam práticas colaborativas no cuidado e assumem relevância para intervenção na dinâmica da vida social das famílias e da própria comunidade, sobretudo sobre os múltiplos fatores que influenciam no processo saúde-doença (Brasil, 2013; Moliner \& Lopes, 2013; Nascimento et al., 2017).

Percebeu-se que o tema relacionado aos transtornos relacionados ao uso de substâncias psicoativas não foi abordado nos discursos dos entrevistados, apresentando de certa forma um desafio da assistência com relação ao cuidado ao usuário de álcool e outras drogas. O uso problemático destas substâncias geralmente é multifatorial que envolve aspectos sociais, familiares, psicológicos, físicos, entre outros, no qual demanda uma qualificação adequada dos profissionais, bem como uma eficiente articulação dos diversos equipamentos sociais e serviços de saúde de referência para o atendimento de casos mais graves (Gerbaldo et al., 2018).

Neste contexto a ESF tem a capacidade de desenvolver ações de prevenção, reabilitação e estratégias de Redução de Danos, no intuito de minimizar as consequências do uso problemático do álcool e outras drogas (Soares et al., 2020).

\subsection{Perspectivas e avanços na produção do cuidado em saúde mental}

Entre as questões que potencializavam a produção do cuidado em SM no âmbito da ESF, destacaram-se a articulação das EqSF com o Nasf-AB e o Centro de Atenção Psicossocial (CAPS), o estabelecimento de vínculos e o desenvolvimento das Práticas Integrativas e Complementares (PICs). Com relação à articulação das EqSF com o Nasf-AB e o CAPS, foi possível identificar, que ela favorece a ampliação do escopo de ações da ESF, possibilitando, entre outros, a oferta de uma escuta qualificada e o acesso a profissionais específicos para além da equipe mínima. Ademais, o Apoio Matricial com o CAPS e a construção do Projeto Terapêutico Singular (PTS) com o Nasf-AB estiveram bastantes presentes. Esses, na maioria das vezes, aconteciam mensalmente e possuíam como objetivo a discussão dos casos mais complexos em SM acompanhados pelas EqSF:

[o que potencializa] A ajuda e colaboração dos CAPS e Nasf-AB (E11).

Então a gente disparou o Nasf-AB [...] o serviço social, me ajudou nesse suporte, nessa costura com a família, a gente fez uma conversa com o CAPS, falando dessa gravidade dessa pessoa, da necessidade que ela tinha de um internamento clínico psiquiátrico [...] (P9).

Agente discute os casos na reunião de equipe e depois de fazer um PTS, fazer o plano terapêutico singular, a gente tenta dar os encaminhamentos devidos. [...]Então a gente tem esse contato muito bom com o CAPS, a gente tem um matriciador que conversa com eles direto 2 a gente faz esse link através do Nasf-AB também, que é o núcleo de Apoio à Saúde da Família, que é esse suporte que dá direto ao CAPS (P2).

A existência do Apoio Matricial realizado pelo CAPS e pelo Nasf-AB, como atuação territorial de interlocução entre os serviços, além de proporcionar a construção de Projetos Terapêuticos Singulares - PTS, tem como objetivo fornecer suporte técnico-pedagógico aos profissionais da ESF, instrumentalizando-os para o cuidado das pessoas em sofrimento psíquico (Jorge et al., 2015). Historicamente, as práticas de SM aconteciam de forma fragmentada e muitas vezes baseadas em transferências de responsabilidades entre os serviços e profissionais da mesma rede de cuidado. No entanto, a mudança na lógica de cuidado através do Apoio Matricial permite a corresponsabilização entre profissionais da rede e possibilita a ampliação do cuidado à pessoa com sofrimento psíquico, fundamental para a efetivação da Reforma Psiquiátrica (Chiaverini et al., 2011).

As atividades de Apoio Matricial contribuem para um processo contínuo e progressivo na ampliação da responsabilidade entre os serviços, e estimula a autonomia das equipes de referência, no caso, a ESF. Essas ações têm objetivo 
de potencializar as intervenções às pessoas com sofrimento psíquico na APS, antes delegadas somente aos serviços especializados de SM. Diante disso, o Apoio Matricial almeja o enfrentamento do problema da fragmentação das condutas e dos saberes, bem como das práticas de cuidado (Lima \& Dimenstein, 2016).

Conforme Gonçalves et al. (2015) o Nasf-AB tem contribuído positivamente no processo de trabalho e na produção do cuidado das EqSF que apoiam, qualificando e aumentando o escopo das ações. A organização e o desenvolvimento do processo de trabalho do Nasf-AB, baseado na clínica ampliada e no apoio matricial, utiliza de ferramentas já amplamente testadas na realidade brasileira, como é o caso do Apoio Matricial, da Clínica Ampliada, do PTS, do Projeto de Saúde no Território e da Pactuação do Apoio (Brasil, 2010).

Contudo, esta estratégia que tenta consolidar-se há cerca de 12 anos, apresentando dificuldades no seu processo de sistematização e organização do trabalho (Tesser, 2017), encara um desafio ainda maior atualmente, após o seu desfinanciamento federal pela portaria 2.979 de 12 de novembro de 2019 (Brasil, 2019), deixando a cargo dos municípios a manutenção das equipes com recursos próprios, correndo o risco de extinção do Nasf-AB em algumas localidades.

Massuda (2020) prevê que esta portaria gere impactos negativos nos serviços da APS a partir da exclusão dos Nasf$\mathrm{AB}$, visto que estes colaboram com o modelo de atenção à saúde preconizada no país.

Enquanto potencialidade, o vínculo foi valorizado como uma ferramenta potente para o desenvolvimento das ações de SM. Essa relação profissional-usuário possibilita a produção de cuidado - através dos atendimentos, visitas, grupos entre outros - na qual os aspectos subjetivos fortalecem o vínculo, a confiança e, consequentemente, a adesão da população ao sistema de saúde. Para tal, é estratégico propiciar escuta e acolhimento de forma mais próxima da população, para assim, identificar ofertas de serviços em potencial e conhecer as reais necessidades de saúde da população adscrita.

O vínculo com as famílias e o conhecimento do contexto familiar. Além da continuidade do acompanhamento pela estratégia Saúde da Família (E28).

A gente tenta primeiro o vínculo... acho que o acolhimento maior é você se mostrar disponível, que justamente no período que a pessoa tá mais vulnerável, é aquele momento que a pessoa tá mais precisando de você chegar junto (P6).

[...] importante se você tem uma porta de acesso qualificada, que conhece essa pessoa em profundidade a partir de um método clínico centrado na pessoa que garanta essa vinculação que é para além de uma vinculação institucional, a vinculação pessoa/pessoa e que tem e que nissose fala de subjetividade, dos afetos, das dores, das delícias da vida dessa pessoa (P9).

O vínculo possibilita a construção de relações afetivas e de confiança entre profissionais e a população que além de potencializar as ações terapêuticas, estimula o processo de corresponsabilização dos usuários no cuidado em saúde (Brasil, 2012). Nesse contexto, o vínculo torna-se duradouro ao passo que o cuidado longitudinal é estabelecido através da relação profissional e a comunidade, considerando aspectos subjetivos e as experiências de vida (Arce, \& Souza, 2014).

Nesse ínterim, a premissa do cuidado em SM está pautada na sensibilidade dos profissionais de saúde frente às necessidades das pessoas com sofrimento psíquico, que por intermédio de um interlocutor, possam falar de conflitos e dificuldades que podem estar relacionados à somatizações e problemas clínicos (Mielke et al., 2009; Brasil, 2013). A escuta e olhar sensíveis permitem fortalecer as relações interpessoais e contribuem para uma visão ampliada do sujeito, considerando suas subjetividades e singularidades. Assim, possibilitam o enfrentamento do estigma e do preconceito relacionados às pessoas 
com transtorno mental, bem como fortalecem a integralidade e equidade no cuidado destas (Albuquerque et al., 2016; Santos \& Santos, 2011).

Vale destacar a importância de atividades de educação permanente que visem despertar nos trabalhadores da APS a sensibilidade para o uso das ferramentas necessárias para a condução terapêutica e discussão dos casos em SM em seus territórios.

As PICs foram constantemente referidas pelos respondentes, demonstrando relevância no cuidado em SM, bem como na promoção da saúde, e prevenção e tratamento de doenças. Algumas ações são desenvolvidas nas próprias unidades de saúde, como é o caso da Terapia Comunitária, e outras em serviços específicos das PICs, sendo encaminhados pelos profissionais da ESF.

É realizada prática integrativa (aurículoterapia) semanal, principalmente para as pessoas que apresentam depressão, transtorno de ansiedade (E9).

[...] e alguns quando têm condições a gente encaminha para as práticas integrativas (PICs) [...] $e$ aí agente vai tentando avaliar qual o perfil daquela pessoa, quais são as possibilidades (P6).

[...] a gente tem uma ação de SM que vem sendo desenvolvida aqui há 10 anos, que é o grupo de terapia comunitária [...] uma roda de conversa que a gente consegue fazer com que as próprias pessoas comecem a pensar sobre as possibilidades de resolução, ou melhor, convivência com os seus problemas, tanto familiares, como psicológicos, sociais, seja ele o que for (P8).

As PICs são sistemas e recursos terapêuticos que envolvem abordagens que buscam estimular os mecanismos naturais de prevenção de agravos e recuperação da saúde por meio de tecnologias eficazes e seguras, dando a ênfase na escuta acolhedora, no desenvolvimento do vínculo terapêutico e na integração do ser humano com o meio ambiente e sociedade (Brasil, 2015).

A Terapia Comunitária é uma ferramenta das PICs, na qual se utiliza de um espaço de acolhimento e compartilhamento de experiências de vida, de sofrimentos psíquicos, de potencialidades e autoajuda comunitária (Barreto, 2008). Trata-se de uma ferramenta potente no cuidado em SM na APS e vem demonstrando grande adesão, visto que sua intervenção acontece na própria comunidade e possibilita o acolhimento e identificação de adoecimentos, que em muitos casos, não necessitam de encaminhamentos para os serviços especializados da RAPS (Araújo et al., 2018).

Outra estratégia de cuidado relatada neste estudo foi a auriculoterapia, uma das práticas da Medicina Tradicional Chinesa que utiliza pontos do pavilhão auricular para o tratamento de diversas desordens do corpo, com efeitos positivos nos aspectos físicos, psíquicos e mentais (Kurebayashi et al., 2012).

\subsection{Desafios para alcançar o cuidado em saúde mental}

No que se refere aos desafios apontados pelos participantes para o desenvolvimento do cuidado em SM na ESF, destacaram-se a fragmentação da RAPS e a fragilidade da promoção de Educação Permanente. A fragmentação da RAPS está presente nos discursos dos dois grupos de entrevistados. Esses referiram estarem inseridos em uma rede fragilizada, com dificuldades de encaminhamentos aos demais pontos de atenção à saúde, carência de recursos humanos e burocratização do fluxo entre os serviços, que repercute diretamente no cuidado oferecido aos usuários. 
[fragilidades] Acesso aos especialistas, acesso ao serviço de urgência quando necessário, fragilidade dos CAPS [...] (E79).

As fragilidades se revelam, quando os projetos terapêuticos, extrapolam o limite de atuação da atenção básica, e envolvem outros níveis de atenção, ou ação de outras secretarias e instituições ou esferas de governo (E42).

Você vai na recepção e marca pelo sistema e para conseguir é difícil, tem paciente que já surtou porque não consegue marcar psiquiatra (P11).

A fragmentação do sistema de saúde é considerada um dos grandes entraves para efetivar o cuidado integral da população. Apesar de existirem normativas que regulamentam a estruturação da RAPS no SUS, essas muitas vezes, não se comunicam de forma adequada e tornam-se isoladas e ineficientes. Uma rede articulada possibilita o acompanhamento longitudinal do usuário, bem como o conhecimento dos profissionais acerca dos encaminhamentos adequados.

De acordo com Júnior (2014) a persistência da fragmentação do SUS tem se apresentado como uma situação hegemônica o tem impedido de se tornar definitivamente um sistema. No caso da RAPS, além da dificuldade dos profissionais em relação ao conhecimento e manejo nas intervenções em SM, percebe-se uma fragilidade da rede que produz uma descontinuidade do cuidado às pessoas com sofrimento psíquico e desarticulação entre serviços e outros setores (Dimenstein et al., 2012, 2018; Rézio et al., 2015).

Outro aspecto identificado como um desafio para o desenvolvimento de ações no campo da SM pelos entrevistados de ambos os grupos foi a percepção do despreparo e a insuficiente capacitação das EqSF para a produção do cuidado para estes casos.

Alguns membros da equipe não se sentem preparados para realizar ações voltadas à SM (E75).

Faltam capacitações para que possamos atuar nesta área (E86).

Não, não tem! Eu nunca tive formação pra isso. Voltada para o paciente, eu desconheço. [...] se teve eu não fui chamada. A gente vê coisa para tuberculose, para Saúde da Mulher, para psiquiatria eu nunca vi não (P11).

A mudança da lógica de cuidado em saúde é um processo contínuo e representa ainda um desafio às EqSF, visto que muitos profissionais possuem uma formação acadêmica baseada no modelo biologicista, centrado na doença. Ademais, o acompanhamento às pessoas com sofrimento psíquico geram inquietações e dificuldades de manejo de profissionais no cotidiano da APS. Assim, se faz necessário que sejam ofertadas ações de Educação Permanente, com vistas a qualificar a produção do cuidado em SM.

Conforme Quinderé (2013) algumas EqSF relatam a falta de instrumentalização para intervir na SM, o que muitas vezes gera encaminhamentos precipitados para os CAPS e outros serviços especializados. Evidências sugerem que parte das EqSF não possuem o devido preparo para o acompanhamento às pessoas com sofrimento psíquico, bem como o desconhecimento da dimensão epidemiológica dos problemas em SM, dificultando, assim, a compreensão do seu papel no cuidado à estas pessoas (Borges \& Teixeira, 2019; Dimenstein et al., 2018; Frateschi \& Cardoso, 2016).

Contudo, é fundamental que os profissionais dos diversos serviços que compõem a RAPS estejam integrados, além dos protocolos existentes, havendo momentos de encontros para discussão de casos e troca de saberes, como por exemplo, a Educação Permanente em Saúde, que contribui para o desenvolvimento do acolhimento, do vínculo e da responsabilização 
(Tanaka, \& Ribeiro, 2009). Fiaramonte et al. (2013) e Campos, Bezerra e Jorge (2018) referem que a ausência de capacitações e atualizações no campo da SM, juntamente com a precária articulação entre os serviços para proporcionar o compartilhamento dos casos e troca de saberes, prejudica o acompanhamento e a qualidade da assistência às pessoas com sofrimento psíquico.

Os profissionais com maior capacidade e preparo no acompanhamento das demandas de SM são aqueles que realizam Educação Permanente. Diante disso, acredita-se que é necessária a sensibilização dos profissionais para uma assistência qualificada e eficaz da pessoa com sofrimento psíquico, tornando-se imprescindível que participem de capacitações profissionais que contribuem para uma melhor tomada de decisões (Gerbaldo et al., 2018).

\section{Considerações Finais}

As práticas de cuidado em SM na APS é um processo em construção e fundamental para a mudança na lógica de cuidado, preconizando assim, o modelo psicossocial como recurso essencial para assistência a pessoa com sofrimento psíquico na comunidade.

Diante dos achados desse estudo, é perceptível que o modelo biomédico ainda está enraizado culturalmente na população e em alguns serviços de saúde. Na realidade estudada, houve grande demanda de usuários que procuram o atendimento para renovação de receitas de psicotrópicos. Este fato torna-se um desafio na prática dos profissionais, que precisam buscar meios para orientar a comunidade acerca dos cuidados direcionados à SM que vai para além do uso do medicamento.

Entretanto, algumas equipes desenvolvem atividades que rompem com a prática medicalizante, havendo a participação de vários profissionais no cuidado em SM. Enquanto potencialidades pode-se mencionar a oferta de atividades em grupo e a utilização das PICs no cuidado em SM na ESF. Tais questões representam avanços na mudança de paradigma assistencial.

A comunicação estabelecida entre a APS e CAPS, articulada pela equipe do Nasf-AB por meio do Apoio Matricial mostrou-se uma ferramenta importante neste estudo, visto que proporciona um espaço de trocas de saberes entre os diversos profissionais da rede e apresenta novas possibilidades de intervenções no cuidado à pessoa com sofrimento psíquico. Contudo, cabe destacar que diante do cenário atual do país, a falta de financiamento federal da APS poderá causar impactos negativos ao modelo do SUS, tornando o acesso do usuário, família e comunidade à assistência individual e coletiva limitado.

Apesar de muitos avanços no cuidado em SM no contexto da APS, alguns desafios foram encontrados. A Educação Permanente apresenta-se ainda incipiente e necessita de um olhar especial dos gestores a fim de priorizar a formação destes profissionais no modelo psicossocial. A rede de serviços, no caso a RAPS, mostrou-se fragmentada, com burocratização do fluxo e baixa integração aos demais serviços especializados de SM, dificultando assim a continuidade do cuidado do usuário com sofrimento psíquico.

Outra problemática que merece ser destacada refere-se às ações relacionadas às pessoas com uso, abuso ou dependência decorrentes ao álcool e outras drogas terem sido pouco relatadas neste estudo. Assim, essas questões merecem ser aprofundadas em novos estudos visto que esta população tem aumentado e frequentemente, encontra-se em contexto de vulnerabilidade.

Espera-se que este estudo contribua para o fomento de estratégias que concretizem uma assistência qualificada e eficaz às pessoas com sofrimento psíquico, ressaltando o envolvimento da gestão na oferta de capacitações para os profissionais da APS. Além disso, estabelecer uma RAPS articulada, com acesso coordenado pela APS, é fundamental para que o acompanhamento às pessoas com sofrimento psíquico seja resolutivo de acordo com a singularidade de cada situação. 


\section{Referências}

Albuquerque, M. C. D. S., Brêda, M. Z., da Costa Maynart, W. H., Silva, D. D. S. D., \& de Medeiros Moura, E. C. (2016). Relacionamento interpessoal entre usuários e profissionais de saúde na atenção psicossocial. Cogitare Enfermagem, 21(3), 01-09. doi: 10.1176/appi.ps.61.3.218.

Amarante, P., \& Nunes, M. D. O. (2018). A reforma psiquiátrica no SUS e a luta por uma sociedade sem manicômios. Ciência \& Saúde Coletiva, 23(6), 20672074. doi: 10.1590/1413-81232018236.07082018

Andrade, A. M., Guimarães, A. M. D., Costa, D. M., Machado, L. D. C., \& Gois, C. F. L. (2014). Visita domiciliar: validação de um instrum ento para registro e acompanhamento dos indivíduos e das famílias. Epidemiologia e Serviços de Saúde, 23, 165-175. doi: 10.5123/S1679-49742014000100016

Araújo, M. Â. M., Girão, J. E. D. P., Souza, K. M. M. D., Esmeraldo, G. R. O. V., Farias, F. L. R. D., \& Souza, Â. M. A. (201 8). A Terapia Comunitária: criando redes solidárias em um Centro de Saúde da Família. Revista Portuguesa de Enfermagem de Saúde Mental, (19), 71-76. doi: 10.19131/rpesm.0204

Arce, V. A. R., \& Sousa, M. F. D. (2014). Práticas de longitudinalidade no âmbito da Estratégia Saúde da Família no Distrito Federal. Cadernos Saúde Coletiva, 22(1), 62-68. doi: 10.1590/1414-462X201400010010

Baker, N., \& Naidu, K. (2020). The Challenges Faced by Mental Health Care Users in a Primary Care Setting: A Qualitative Study. Community Mental Health Journal, 1-9. doi: 10.1007/s10597-020-00647-y

Balsanelli, A. P., \& Cunha, I. C. K. O. (2006). Liderança no contexto da enfermagem. Revista da Escola de Enfermagem da USP, 40(1), 117-122. doi: 10.1590/S0080-62342006000100017

Bardin, L. (2011). Análise de conteúdo. Lisboa, Portugal: Edições 70.

Barreto, A. D. P. (2008). Terapia Comunitária Passo a Passo. $3^{a}$. Edição. Fortaleza: Gráfica LCR.

Barros, S., Nóbrega, M. D. P. S. D. S., Santos, J. C. D., Fonseca, L. M. D., \& Floriano, L. S. M. (2019). Saúde mental na ate nção primária: processo saúdedoença, segundo profissionais de saúde. Revista Brasileira de Enfermagem, 72(6), 1609-1617. doi: 10.1590/0034-7167-2018-0743

Borges, N. S., \& Teixeira, L. A. (2019). Integração entre a Estratégia Saúde da Família e o Centro de Atenção Psicossocial. Revista Enfermagem Contemporânea, 8(1), 59-71. doi: 10.17267/2317-3378rec.v8i1.2209

Brasil. (2017). Portaria n ${ }^{\circ}$ 2.436, de 21 de setembro de 2017. Aprova a Política Nacional de Atenção Básica, estabelecendo a revisão de diretrizes para a organização da Atenção Básica, no âmbito do Sistema Único de Saúde (SUS). Diário Oficial da União, 183(1).

Brasil. (2019). Institui o Programa Previne Brasil, que estabelece novo modelo de financiamento de custeio da Atenção Primária à Saúde no âmbito do Sistema Único de Saúde (SUS).

Brasil. Ministério da Saúde (2010). Diretrizes do NASF: núcleo de apoio à saúde da família. Cadernos de Atenção Básica, (27).

Brasil. Ministério da Saúde (2011). Portaria nº 3.088, de 23 de dezembro de 2011. Institui a Rede de Atenção Psicossocial para pessoas com sofrimento ou transtorno mental e com necessidades decorrentes do uso de crack, álcool e outras drogas, no âmbito do Sistema Único de Saúde (SUS). Diário Oficial da União.

Brasil. Ministério da Saúde. (2015). Política Nacional de Práticas Integrativas e Complementares no SUS: atitude de ampliação de acesso.

Brasil. Ministério da Saúde. Secretaria de Atenção à Saúde. (2013). Cadernos de atenção básica: Saúde Mental (34).

Brasil. Ministério da Saúde. Secretaria de Atenção à Saúde. Departamento de Atenção Básica. (2012). Caderno de atenção domiciliar.

Brasil. Resolução n. 466. (2012). Diretrizes e normas regulamentadoras de pesquisas envolvendo seres humanos.

Brito, G. E. G. D., Mendes, A. D. C. G., \& Santos Neto, P. M. D. (2018). O objeto de trabalho na Estratégia Saúde da Família. Interface-Comunicação, Saúde, Educação, 22(64), 77-86. doi: 10.1590/1807-57622016.0672

Campos, D. B., Bezerra, I. C., \& Jorge, M. S. B. (2018). Tecnologias do cuidado em saúde mental: práticas e processos da Atenção Primária. Revista Brasileira de Enfermagem, 71, 2101-2108. doi: 10.1590/0034-7167-2017-0478

Chiaverini, D.H. (2011). Guia prático de matriciamento em saúde mental. Brasília, DF: Ministério da Saúde.

Dimenstein, M., Amorim, A. K. A., Leite, J., Siqueira, K., Gruska, V., Vieira, C., ... \& Bezerril, M. C. (2012). O atendimento da crise nos diversos componentes da rede de atenção psicossocial em Natal/RN. Revista Polis e Psique, 2(3), 98. doi:10.22456/2238-152X.40323

Dimenstein, M., Macedo, J. P., Gomes, M., Da Silva, T. M., \& De Abreu, M. M. (2018). A saúde mental e atenção psicossocial: Regionalização e gestão do cuidado integral no SUS. Salud \& Sociedad, 9(1), 070-085. doi: 10.22199/S07187475.2018.0001.00004

Fioramonte, A., Bressan, B. F., da Silva, E. M., do Nascimento, G. L., \& Buriola, A. A. (2013). Cuidado à pessoa com transtorno mental e sua família: atuação do enfermeiro na ESF. Ciência, Cuidado e Saúde, 12(2), 315-322. doi: 10.4025/cienccuidsaude.v12i2.20362

Frateschi, M. S., \& Cardoso, C. L. (2016). Práticas em saúde mental na atenção primária à saúde. Psico,47(2), 159-168. doi:10.15448/19808623.2016.2.22024

Gerbaldo, T. B., Arruda, A. T., Horta, B. L., \& Garnelo, L. (2018). Avaliação da organização do cuidado em saúde mental na atenção básica à saúde do Brasil. Trabalho, Educação e Saúde, 16(3), 1079-1094. doi: 10.1590/1981-7746-sol00150 
Giovanella, L., de Mendonça, M. H. M., Buss, P. M., Fleury, S., Gadelha, C. A. G., Galvão, L. A. C., \& dos Santos, R. F. (2019). De Alma-Ata a Astana. Atenção primária à saúde e sistemas universais de saúde: compromisso indissociável e direito humano fundamental. Cadernos de Saúde Pública, 35(3), e00012219. doi: 10.1590/0102-311X00012219

Gonçalves, R. M. D. A., Lancman, S., Sznelwar, L. I., Cordone, N. G., \& Barros, J. D. O. (2015). Estudo do trabalho em núcleos de apoio à saúde da família (NASF), São Paulo, Brasil. Revista Brasileira de Saúde Ocupacional, 40(131), 59-74. doi: 10.1590/0303-7657000078013

Gryschek, G., \& Pinto, A. A. M. (2015). Saúde Mental: como as equipes de Saúde da Família podem integrar esse cuidado na Aten ção Básica? Ciência \& Saúde Coletiva, 20, 3255-3262. doi: 10.1590/1413-812320152010.13572014

Jorge, M. S. B., Diniz, A. M., Lima, L. L. D., \& Penha, J. C. D. (2015). Apoio matricial, projeto terapêutico singular e produção do cuidado em saúde mental. Texto \& Contexto-Enfermagem, 24(1), 112-120. doi: 10.1590/0104-07072015002430013

Junior, H. M. M. (2014). Redes de Atenção à Saúde: rumo à integralidade. Divulgação em saúde para debate [on-line], 52, 15-37.

Kurebayashi, L. F. S., Gnatta, J. R., Borges, T. P., \& da Silva, M. J. P. (2012). Aplicabilidade da auriculoterapia para redu zir estresse e como estratégia de coping em profissionais de enfermagem. Revista Latino-Americana de Enfermagem, 20(5), 980-987. doi: 10.1590/S0104-11692012000500021

Lima, M., \& Dimenstein, M. (2016). O apoio matricial em saúde mental: uma ferramenta apoiadora da atenção à crise. Interface-Comunicação, Saúde, Educação, 20(58), 625-635. doi: 10.1590/1807-57622015.0389

Luitel, N. P., Jordans, M. J. D., Subba, P., \& Komproe, I. H. (2020). Perception of service users and their caregivers on primary care-based mental health services: a qualitative study in Nepal. BMC Family Practice, 21(1), 1-11. doi: 10.1186/s12875-020-01266-y

Macinko, J., \& Mendonça, C. S. (2018). Estratégia Saúde da Família, um forte modelo de Atenção Primária à Saúde que traz resultados. Saúde em Debate, 42(spe1), 18-37. doi: 10.1590/0103-11042018s102

Maia, J. L. F. (2020). Saúde Mental Pública no Brasil: Interfaces com a Atenção Básica à Saúde. Cadernos Brasileiros de Saúde Mental/Brazilian Journal of Mental Health, 12(33), 01-15. ISSN 1984-2147

Massuda, A. (2020). Mudanças no financiamento da Atenção Primária à Saúde no Sistema de Saúde Brasileiro: avanço ou retrocesso? Ciência \& Saúde Coletiva, 25(4), 1181-1188. doi: 10.1590/1413-81232020254.01022020

Matuda, C. G., da Silva Pinto, N. R., Martins, C. L., \& Frazão, P. (2015). Colaboração interprofissional na estratégia saúde da família: implicações para a produção do cuidado e a gestão do trabalho. Ciência \& Saúde Coletiva, 20(8), 2511-2521. doi: 10.1590/1413-81232015208.11652014

Melo, S. C. C., Santana, R. G. D., Santos, D. C. D., \& Alvim, N. A. T. (2013). Práticas complementares de saúde e os desafios de sua aplicabilidade no hospital: visão de enfermeiros. Revista Brasileira de Enfermagem, 66(6), 840-846. doi: 10.1590/S0034-71672013000600005

Mielke, F. B., Kantorski, L. P., Jardim, V. M. D. R., Olschowsky, A., \& Machado, M. S. (2009). O cuidado em saúde mental no CAPS no entendimento dos profissionais. Ciência \& Saúde Coletiva, 14(1), 159-164. doi: 10.1590/S1413-81232009000100021

Minozzo, F., Kammzetser, C. S., Debastiani, C., Fait, C. S., \& Paulon, S. M. (2012). Mental health groups in primary health care. Fractal: Revista de Psicologia, 24(2), 323-340. doi: 10.1590/S1984-02922012000200008

Moliner, J. D., \& Lopes, S. M. B. (2013). Saúde mental na atenção básica: possibilidades para uma prática voltada para a ampliação e integralidade da saúde mental. Saúde e Sociedade, 22(4), 1072-1083. doi: 10.1590/S0104-12902013000400010

Moreira, M. I. B., \& Onocko-Campos, R. T. (2017). Ações de saúde mental na rede de atenção psicossocial pela perspectiva dos usuários. Saúde e Sociedade, 26(2), 462-474. doi: 10.1590/s0104-12902017171154

Nascimento, V. F., Terças, A. C. P., Hattori, T. Y., Cabral, J. F., Gleriano, J. S., Borges, A. P., ...\& da Silva, R. G. M. (2017). Percepção de agentes comunitários de saúde sobre visita domiciliária após aperfeiçoamento em saúde da família. Revista de APS, 20(3) 392-402. doi: 10.34019/18098363.2017.v20.15966

Neto, D. C. (2017). A (des) medicalização na atenção primária: o surgimento de um novo cenário na saúde pública. Revista Científica FAGOC-Saúde, 1(2), 914.

Organização Mundial de Saúde (2010). World health statistics 2010. Geneva: World Health Organization.

Organização Mundial de Saúde (2016). mhGAP intervention guide for mental, neurological and substance use disorders in non-specialized health settings: mental health Gap Action Programme (mhGAP). Geneva: World Health Organization.

Organização Pan-Americana da Saúde (OPAS). (2018a) La carga de los transtornos mentales en la Región de las Américas, Brasília: Organização PanAmericana de Saúde.

Organização Pan-Americana da Saúde (OPAS). (2018b). Relatório 30 anos de SUS, que SUS para 2030? Brasília: Organização Pan-Americana de Saúde. Paulon, S., Neves, R., Dimenstein, M., Nardi, H., Bravo, O., Galvão, V. A. B. D. M., ... \& Figueró, R. (2013). A saúde mental no contexto da Estratégia Saúde da Família no Brasil. Psicologia para América Latina, (25), 24-42. ISSN 1870-350X

Peduzzi, M., Norman, I. J., Germani, A. C. C. G., Silva, J. A. M. D., \& Souza, G. C. D. (2013). Educação interprofissional: formação de profissionais de saúde para o trabalho em equipe com foco nos usuários. Revista da Escola de Enfermagem da USP, 47(4), 977-983. doi: 10.1590/S0080-623420130000400029

Quinderé, P. H. D., Jorge, M. S. B., Nogueira, M. S. L., Costa, L. F. A. D., \& Vasconcelos, M. G. F. (2013). Acessibilidade e resolubilidade da assistência em saúde mental: a experiência do apoio matricial. Ciência \& Saúde Coletiva, 18, 2157-2166. doi: 10.1590/S1413-81232013000700031 
Research, Society and Development, v. 10, n. 2, e45210212674, 2021

(CC BY 4.0) | ISSN 2525-3409 | DOI: http://dx.doi.org/10.33448/rsd-v10i2.12674

Rézio, L. D. A., Moro, T. N., Marcon, S. R., \& Fortuna, C. M. (2015). Contribuições do PET-Saúde/Redes de Atenção Psicossocial à Saúde da Família. Interface-Comunicação, Saúde, Educação, 19(1), 793-803. doi: 10.1590/1807-57622014.0673

Rodriguez, J. J. (2010). Estratégia e plano de ação sobre saúde mental para a região das Américas. Brazilian Journal of Psychiatry, 32(4), 341-342. doi: $10.1590 / \mathrm{S} 1516-44462010000400004$

Santos, I. M. V. D., \& Santos, A. M. D. (2011). Acolhimento no Programa Saúde da Família: revisão das abordagens em periódicos brasileiros. Revista de SaludPública, 13(4), 703-716. doi: 10.1590/S0124-00642011000400015

Saunders, B., Sim, J.,Kingstone, T., Baker, S., Waterfield, J., Bartlam, B., ... \&Jinks, C. (2018). Saturation in qualitative research: exploring its conceptualization and operationalization. Quality \& Quantity, 52(4), 1893-1907. doi: 10.1007/s11135-017-0574-8

Serapioni, M., \& Tesser, C. D. (2019). O Sistema de Saúde brasileiro ante a tipologia internacional: uma discussão prospectiva e inevitável. Saúde em Debate, 43(spe5), 44-57. doi: 10.1590/0103-11042019S504

Soares, N. S. A., Fernandes, M. A., Ribeiro, H. K. P., Rocha, D. D. M., \& Ribeiro, Í. A. P. (2020). Redução de danos na atenção primária à saúde: revisão integrativa das estratégias assistenciais. Revista da Escola de Enfermagem da USP, 54. doi: 10.1590/s1980-220x2018051803591

Souza, Â. C., Amarante, P. D., \& Abrahão, A. L. (2019). Inclusão da saúde mental na atenção básica à saúde: estratégia de cuidado no território. Revista Brasileira de Enfermagem, 72(6), 1677-1982. doi: 10.1590/0034-7167-2018-0806

Tanaka, O. Y., \& Ribeiro, E. L. (2009). Ações de saúde mental na atenção básica: caminho para ampliação da integralidade da atenção. Ciência \& Saúde Coletiva, 14(2), 477-486. doi: 10.1590/S1413-81232009000200016

Tesser, C. D. (2017). Núcleos de Apoio à Saúde da Família, seus potenciais e entraves: uma interpretação a partir da atenção primária à saúde. InterfaceComunicação, Saúde, Educação, 21(62), 565-578. doi: 10.1590/1807-57622015.0939

Tesser, C. D., Neto, P. P., \& de Campos, G. W. (2010). Acolhimento e (des) medicalização social: um desafio para as equipes de saúde da família. Ciência \& Saúde Coletiva, 15(3), 3615-3624. doi: 10.1590/S1413-81232010000900036

Wenceslau, L. D., \& Ortega, F. (2015). Salud mental en la atención primaria y Salud Mental Global: perspectivas internacionales y el escenario brasileño. Interface-Comunicação, Saúde, Educação, 19(55), 1121-1132. doi: 10.1590/1807-57622014.1152

Wetzel, C., Kohlrausch, E. R., Pavani, F. M., Batistella, F. S., \& Pinho, L. B. D. (2018). Análise sobre a formação interprofissional em serviço em um Centro de Atenção Psicossocial. Interface-Comunicação, Saúde, Educação, 22, 1729-1738. doi: 10.1590/1807-57622017.0664

Zanella, M., Luz, H. H. V., Benetti, I. C., \&Roberti Junior, J. P. (2016). Medicalização e saúde mental: Estratégias alternativas. Revista Portuguesa de Enfermagem de Saúde Mental, (15), 53-62. doi: 10.19131/rpesm.0132 